\title{
The fractal dimensions of the spatial distribution of young open clusters in the solar neighbourhood
}

\author{
R. de la Fuente Marcos and C. de la Fuente Marcos
}

\author{
Suffolk University Madrid Campus, C/ Viña 3, 28003 Madrid, Spain \\ e-mail: raul@galaxy.suffolk.es
}

Received 21 November 2005 / Accepted 26 January 2006

\begin{abstract}
Context. Fractals are geometric objects with dimensionalities that are not integers. They play a fundamental role in the dynamics of chaotic systems. Observation of fractal structure in both the gas and the star-forming sites in galaxies suggests that the spatial distribution of young open clusters should follow a fractal pattern, too.

Aims. Here we investigate the fractal pattern of the distribution of young open clusters in the Solar Neighbourhood using a volumelimited sample from WEBDA and a multifractal analysis. By counting the number of objects inside spheres of different radii centred on clusters, we study the homogeneity of the distribution.

Methods. The fractal dimension $D$ of the spatial distribution of a volume-limited sample of young open clusters is determined by analysing different moments of the count-in-cells. The spectrum of the Minkowski-Bouligand dimension of the distribution is studied as a function of the parameter $q$. The sample is corrected for dynamical effects.

Results. The Minkowski-Bouligand dimension varies with $q$ in the range $0.71-1.77$, therefore the distribution of young open clusters is fractal. We estimate that the average value of the fractal dimension is $\langle D\rangle=1.7 \pm 0.2$ for the distribution of young open clusters studied.

Conclusions. The spatial distribution of young open clusters in the Solar Neighbourhood exhibits multifractal structure. The fractal dimension is time-dependent, increasing over time. The values found are consistent with the fractal dimension of star-forming sites in other spiral galaxies.
\end{abstract}

Key words. methods: statistical - stars: formation - open clusters and associations: general - solar neighbourhood

\section{Introduction}

Fractals are geometric objects with dimensionalities that are not integers. They play a fundamental role in the dynamics of chaotic systems (Mandelbrot 1982, 1985). Fractals are often used to model hierarchical, non-homogeneous structures in various areas of Astrophysics, in particular the distribution of matter in the Universe. Interstellar gas, giant molecular clouds, and stars in galaxies are observed to have a fractal structure ranging from subparsec scales to several kiloparsecs scales (see Elmegreen \& Elmegreen 2001, and references therein). Starforming regions and eventually stars are formed out of this gas. Star-forming complexes are ubiquitous in spiral and irregular galaxies and are probably the fundamental scale for star formation there. Star formation is an essentially chaotic process; therefore, star-forming regions usually exhibit a very clumpy, filamentary nature. Simulations (e.g. Kroupa et al. 2001) suggest that open clusters form as the nuclei of star-forming complexes.

The observation of fractal structure in both the gas and the star-forming sites (see review by Elmegreen et al. 2000) in galaxies suggests that the spatial distribution of young open clusters should follow a fractal pattern, too. Here we show evidence of such a fractal pattern in a volume-limited sample of young open clusters in the Solar Neighbourhood. This paper is organised as follows. In Sect. 2 we briefly present the data analysed. The spatial distribution of young open clusters is studied in Sect. 3. In Sect. 4 we calculate the fractal dimension. In Sect. 5 we compare our results with those obtained by other authors. Finally, in Sect. 6 we discuss our results.

\section{Data}

In order to evaluate the fractal dimension of young open clusters in the Milky Way, we have to construct a representative sample of open clusters. In principle, the optimal approach will be to assemble an age-limited, volume-limited open cluster sample in the solar vicinity. The Solar Neighbourhood is defined as a volume centred on the Sun that is much smaller than the overall size of the Milky Way galaxy and yet large enough to contain a statistically useful sample of stars (see, e.g., Binney \& Tremaine 1987). The appropriate size of the volume depends on which stars or objects (in this case) are going to be investigated: for white dwarfs, which are both common and faint, it may consist of a sphere of radius $10 \mathrm{pc}$ centred on the Sun, while for the bright but rare $\mathrm{O}$ and $\mathrm{B}$ stars, the Solar Neighbourhood may be considered to extend as far as $1 \mathrm{kpc}$ from the Sun. In our analysis we consider $2.5 \mathrm{kpc}$ in order to retain a statistically significant sample of young open clusters (see below).

The positions of the sample of young open clusters in the Solar Neighbourhood were taken from the Open Cluster Database (WEBDA) developed by Mermilliod (1988a,b, 1992a,b, 1993, 1995, 1996) and maintained by Paunzen (Paunzen \& Mermilliod 2005). The latest update of the Open Cluster Database (December 2005) includes 1747 open clusters with ages for 959 objects $(54.9 \%)$. In this database we have found 336 objects $(35 \%)$ with age $\leq 60$ Myr. Out of this sample, 267 objects are located within $2.5 \mathrm{kpc}$ from the Sun. On the other hand, the Sun appears to be displaced from the Galactic plane. Bonatto et al. (2006) have found that its location is 


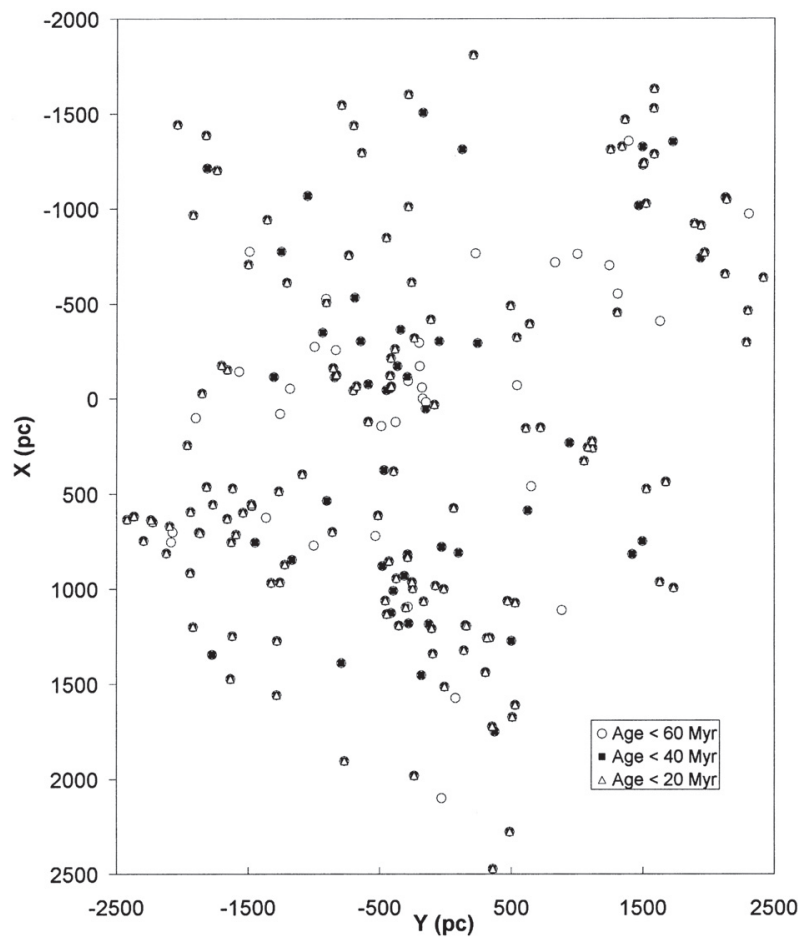

Fig. 1. Distribution of young open clusters for the studied sample in Heliocentric Galactic rectangular coordinates. Positions have been calculated using data from a recent update (December 30, 2005) of WEBDA (Paunzen \& Mermilliod 2005). Three age groups are considered (20, 40, and 60 Myr old open clusters).

$z_{\odot}=14.8 \pm 2.4 \mathrm{pc}$ above the Galactic plane. Although the distribution of young open clusters in terms of Galactic latitude shows that most of them are tightly concentrated close to the Galactic plane, it is obvious that the number of young open clusters located at Southern latitudes is significantly higher. If we calculate the vertical position $z$ of all the young open clusters in our initial sample using Galactic longitude, latitude, and distance from the Sun, we obtain an average vertical position, $\langle z\rangle \sim-22 \mathrm{pc}$. Taking this asymmetry into account, we have selected a subsample with $z$ in the range $(-97,53) \mathrm{pc}$. This is equivalent to a scale height for the sample of $75 \mathrm{pc}$. Therefore, the volume occupied by our final sample of 209 objects is $2.94 \mathrm{kpc}^{3}$. The average vertical position for the final sample is $\langle z\rangle=-15.8 \mathrm{pc}$, which is consistent with the value quoted by Bonatto et al. (2006). Figure 1 suggests that the spatial distribution of young open clusters in the Solar Neighbourhood is rather filamentary in nature, although it shows clear signs of clumpiness, as the hundreds of parsecs wide star-forming complexes include many open clusters in a relatively small region. The filamentary nature is mainly associated to the presence of the Saggitarius arm (towards the Galactic Centre, left-lower part of the figure) and the Perseus arm (towards the Galactic Anticentre, right-upper part of the figure). The inter-arm structure traced by the young open clusters appears to be filamentary, too (the Orion spur). In the next section, we analyse the cumulative distance function and in Sect. 4, we quantify the pattern of the distribution of young open clusters using Mandelbrot's theory of fractals.

\section{The spatial distribution}

In order to assess whether the spatial distribution of young open clusters is fractal, we first examine the cumulative distance distribution: $N(<d) \propto d^{\beta}$, where $N(<d)$ is the cumulative number

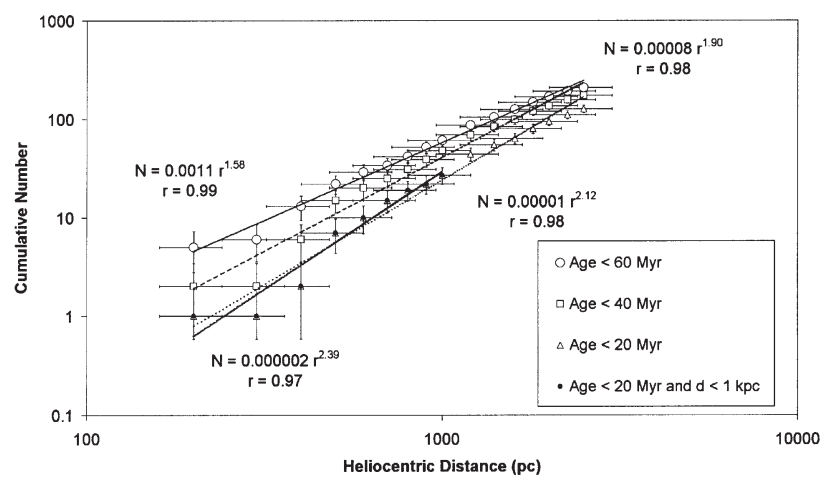

Fig. 2. Cumulative distance distribution for the three age groups in the sample of 209 open clusters. There is evidence of incompleteness at the high distance end and the results for low distances $(d<250 \mathrm{pc})$ are not reliable due to low number statistics caused by the Local Bubble (see the text).

of objects with a distance shorter than $d$ and $\beta$ is the exponent of the (assumed) power-law distribution. As in Fig. 1, three age groups are considered: 20 (126 clusters), 40 (173 clusters), and $60 \mathrm{Myr}$ (209 clusters). If we represent the cumulative distribution function for distances (see Fig. 2) for the three age groups, the exponent of the power-law fit is probably related to the fractal dimension. The error bars in $N$ have been estimated from a Poisson distribution with mean error $\sqrt{N}$; the error bars in distance assume a $20 \%$ standard deviation that is characteristic of open clusters. This is a slightly pessimistic assumption, as the errors strongly depend on the actual distance (the closer, the more accurate) but also on the interstellar extinction towards the cluster that for young open clusters is difficult to calculate. The exponent of the power-law gives $\beta=1.58 \pm 0.04$ for the young opencluster sample in the Solar Neighbourhood (age < $60 \mathrm{Myr}$ ). The small error quoted is the error in the slope. The product-moment correlation coefficient, or Pearson's $r$ is 0.99 .

The distribution analysed in Fig. 2 presents two main problems: the number of young open clusters within $250 \mathrm{pc}$ from the Sun is rather low and for young open clusters beyond $2 \mathrm{kpc}$, the effects of the sample's incompleteness begin to be nonnegligible. The first effect is the result of the presence of the Local Bubble. The Local Bubble region is a neutral, low density interstellar cavity in the galactic plane that surrounds the Sun with radii between 65-250 pc (Sfeir et al. 1999; Lehner et al. 2003). The cavity is surrounded by a dense neutral-gas boundary or wall, and it encloses the Hyades open cluster and the Arcturus group. The Local Bubble contains about 200000 stars and was probably carved out by multiple supernova explosions that occurred several hundred thousand years ago in the Scorpius-Centaurus association, a rich cluster of young stars (Maíz Apellániz 2001; Berghöfer \& Breitschwerdt 2002). If we repeat the calculations for a subsample in the age range $<40 \mathrm{Myr}$ (see Fig. 2), we obtain an exponent of $\beta=1.90 \pm 0.09$ for the young open-cluster sample in the Solar Neighbourhood. In this case the correlation coefficient is 0.98 .

In our selection criteria for the final sample we have tried to minimize the role of dynamical effects such as open cluster disruption or radial/azimuthal drift (orbital diffusion), as well as incompleteness. However, available observational data suggests that about $60-70 \%$ of the open clusters are destroyed within 20 Myr (de la Fuente Marcos \& de la Fuente Marcos 2004) of formation. To correct our sample for this effect, we have considered the subsample of open clusters younger than $20 \mathrm{Myr}$ (126 objects) and yet another subsample correcting the 
previous one from the effects of incompleteness $(d<1.0 \mathrm{kpc}$, 58 objects). In this age range and under quiescent star formation, less than $10 \%$ of newly-formed open clusters have been disrupted as a result of dynamical processes (de la Fuente Marcos $\&$ de la Fuente Marcos 2004). The analysis of both subsamples is summarised in Fig. 2. The exponent for the first subsample is $\beta=2.12 \pm 0.10$, with a correlation coefficient of 0.98 . The final value of the exponent corrected for all effects (second subsample) is $\beta=2.38 \pm 0.23$ with a correlation coefficient of 0.97 .

On the other hand, the distribution of clusters within a thin disk, $150 \mathrm{pc}$ thick with radius $2.5 \mathrm{kpc}$, is more or less like a plane, and the radial number density can be defined as:

$\sigma=\frac{N(r<d)}{\pi r^{2}}$

On the other hand, the radial number density can also be described by a power law:

$\sigma \propto r^{-\alpha}$

Taking both equations into account, we can also write:

$N(r<d) \propto r^{2-\alpha}$.

In our case,

i) for the corrected sample (age $<20 \mathrm{Myr}$ and distance $<1 \mathrm{kpc}$ ) $\alpha=-0.39 \pm 0.23$, therefore the space density decreases slightly over distance;

ii) for clusters younger than $20 \mathrm{Myr}, \alpha=-0.12 \pm 0.10$, which is compatible with constant density;

iii) for clusters younger than $40 \mathrm{Myr}, \alpha=0.10 \pm 0.09$, which is again compatible with constant density;

iv) for the entire sample, $\alpha=0.42 \pm 0.04$.

\section{The fractal dimension}

A fractal point distribution can be described in terms of its fractal dimension. Many apparently different definitions of dimensionality are currently used in nonlinear dynamics. In general, these may all give different numerical values for the dimensionality, although in some cases the numbers are close. To increase the difficulties, the term fractal dimension is used rather indiscriminately. In the present work we adopt the working definition and procedure described by Yadav et al. (2005). These authors have carried out a multifractal analysis of the galaxy distribution in the Sloan Digital Sky Survey Data Release One to conclude that the galaxy distribution is homogeneous on scales larger than 60-70 $h^{-1} \mathrm{Mpc}$.

Following Yadav et al. (2005), let us consider a finite distribution of $N$ young open clusters, labelling the young clusters from 1 to $N$, and using $\boldsymbol{x}_{i}$ and $\boldsymbol{x}_{j}$ to denote the heliocentric coordinates of the $i$ th and $j$ th young open clusters, respectively. Then the number of other young open clusters within a sphere of radius $r$ centred on the $i$ th young cluster is given by the expression:

$n_{i}(r)=\sum_{j=1, j \neq i}^{N} \Theta\left(r-\left|\boldsymbol{x}_{i}-\boldsymbol{x}_{j}\right|\right)$,

where $\Theta(x)$ is the Heaviside function defined such that $\Theta(x)=0$ for $x<0$ and $\Theta(x)=1$ for $x \geq 0$. This summation is over the whole set of young open clusters with coordinates $\boldsymbol{x}_{j}, j \neq i$. If we choose $M$ different young open clusters as centres to evaluate the previous expression and calculate the average

$C_{2}(r)=\frac{1}{M(N-1)} \sum_{i=1}^{M} n_{i}(r)$,

then we obtain the probability of finding a young open cluster within a sphere of radius $r$ centred on another young open cluster. This summation is over a subset of $M$ young clusters, taken as centres, with coordinates $\boldsymbol{x}_{i}$. By taking only $M$ young clusters as centers we allow for the effect of the finiteness of the sample. As $r$ goes to zero, $C_{2}(r)$ can be described as a power-law function of the radius of the sphere, $C_{2}(r)=k r^{D_{2}}$, so then the exponent $D_{2}$ can be identified with the fractal dimension of the young open-cluster sample distribution. For computational purposes it is more convenient to use the form: $\log \left(C_{2}(r)\right)=K+D_{2} \log (r)$, where $K$ is a constant. In fact, $C_{2}(r)$ is related to the two-point correlation function described by the expression:

$\xi(r)=\left(\frac{r}{r_{\mathrm{o}}}\right)^{\gamma}$.

Within this framework, $D_{2}$ is the two-point correlation dimension, $C_{2}(r)$ is closely related to the volume integral of $\xi(r)$, and $D_{2}=2-\gamma$ on scales $r<r_{0}$. In our case, the fractal dimension is necessarily $\leq 2$ for an embedding space of dimension two; when $D_{2}$ is different from two, the distribution is fractal. The disk has a radius of $2.5 \mathrm{kpc}$ but a vertical scale of $150 \mathrm{pc}$, therefore it is almost a plane. The fractal dimension matches the Euclidean dimension for a random spatial distribution in a plane. If the plane approximation is not exact then a small deviation is expected with the maximum value of the fractal dimension slightly over 2 .

Unfortunately, the two-point correlation is not enough to provide a statistically complete description of the properties of the distribution of young open clusters. On the other hand, the full statistical quantification of a fractal distribution cannot be based on one scaling index. In order to provide an exhaustive description of the distribution of young open clusters in the Solar Neighbourhood, higher-order correlations have to be studied. The use of the $(q-1)$ th moment of $n_{i}(r)$ enables the generalization of the two-point correlation dimension as the Minkowski-Bouligand dimension, $D_{q}$ given by $C_{q}(r)=$ $k r^{(q-1) D_{q}}$, where

$C_{q}(r)=\frac{1}{M(N-1)} \sum_{i=1}^{M}\left(n_{i}(r)\right)^{q-1}$

The Minkowski-Bouligand dimension is also called the generalised fractal dimension (see, e.g., Grassberger 1983; Grassberger \& Procaccia 1983). In general, the scaling behaviour of $C_{q}(r)$ depends on the length-scale. A monofractal is characterised by a single value of the Minkowski-Bouligand dimension, independent of $q$. Variations of the Minkowski-Bouligand dimension with $q$ are typical of multifractal distributions. In multifractals, the whole spectrum of singularity exponents is required to accurately describe their geometrical properties (Falconer 1990). The positive values of $q$ give more importance to the regions of the Milky Way disk where the number density of young open clusters (star-forming complexes) is higher, whereas the negative values of $q$ favour the contribution of underdense zones (the gaps or interarm regions). In principle, the Minkowski-Bouligand dimension characterises the scaling of interarm regions for $q<0$ and describes the 


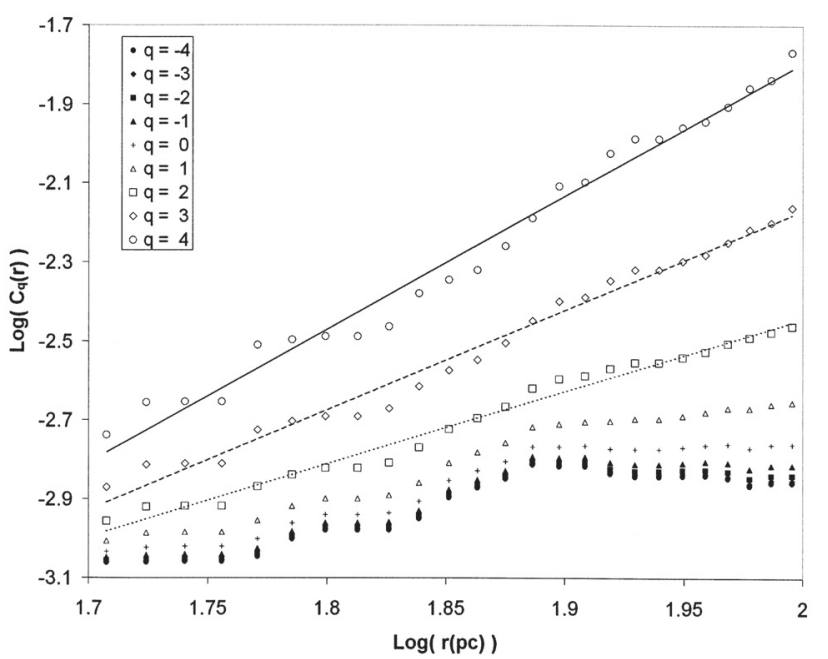

Fig. 3. $C_{q}(r)$ for all the values of $q$ studied for the full subsample of 209 young open clusters.

scaling behaviour of the young open cluster distribution within star-forming complexes for $q>0$ ( $D_{1}$ corresponds to the boxcounting dimension or information dimension and for $q=0 \mathrm{we}$ have the Hausdorff fractal dimension). A fractal dimension of 3 indicates spatial randomness in a three-dimensional space, and a value of 2 indicates spatial randomness in a two-dimensional space. A fractal dimension of 0 would mean complete clumping, with all the objects concentrated at a single point.

Using the method outlined above, we analysed the various subsamples studied in the previous section. For each young open cluster we considered a sphere of radius $r$ centred on the cluster and counted the number of other clusters to determine $n_{i}(r)$. The characteristic radius is in the range $50-100 \mathrm{pc}$, and the sphere is always within the boundaries defined by the sample. The values of $n_{i}(r)$ calculated using different clusters as centres were then averaged to calculate $C_{q}(r)$, after which we draw the log-log plot of $C_{q}(r)$ versus $r$, estimate the value of the slope of the graph $\tau(q)$ with a linear regression, and obtain a dimension given by

$D_{q}=\frac{\tau(q)}{q-1}$

$D_{1}=\lim _{q \rightarrow 1} D_{q}$.

This method assumes $C_{q}(r) \propto r^{(q-1) D_{q}}$, although for computational purposes it is more convenient to use the form

$\log \left(C_{q}(r)\right)=K+(q-1) D_{q} \log (r)$,

where $K$ is a constant. The generalised dimensions given by Eq. (8) provide a spectrum. This spectrum of dimensions characterises the multifractal. It can be proved (Paladin \& Vulpiani 1987) that the generalised dimensions are monotonically decreasing. In order to keep the spheres within the boundaries of the samples, the number of centres decrease when $r$ increases.

Figure 3 shows the $\log -\log$ plot of $C_{q}(r)$ for values of $q$ in the range $(-4,4)$ in steps of 1 for the 209 objects in the first sample (age $<60$ Myr). The slope gives the Minkowski-Bouligand dimension for each value of $q$. According to Eq. (8), the values of the slope for $q \leq 0$ give unphysical results $\left(D_{q}<0\right)$, so we focus our attention on $q>0$. The slope ranges from $1.85 \pm 0.05$ for $q=2$ to $3.38 \pm 0.10$ for $q=4$. The correlation dimension, $D_{2}$, is $1.85 \pm 0.05, D_{3}=1.26 \pm 0.07$ and $D_{4}=1.13 \pm 0.10$. The correlation coefficient is 0.99 .

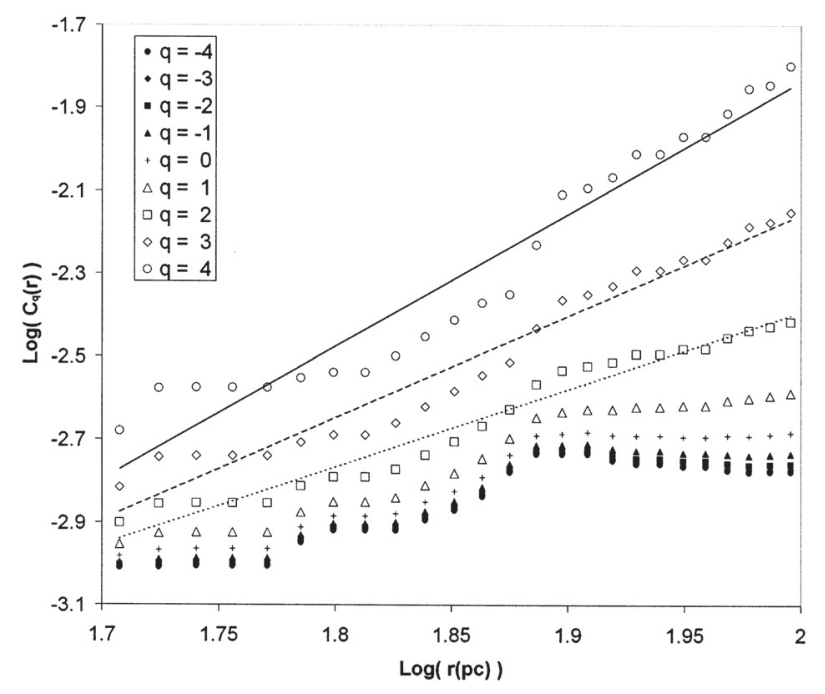

Fig. 4. $C_{q}(r)$ for age group $<40 \mathrm{Myr}$ old of 173 open clusters.

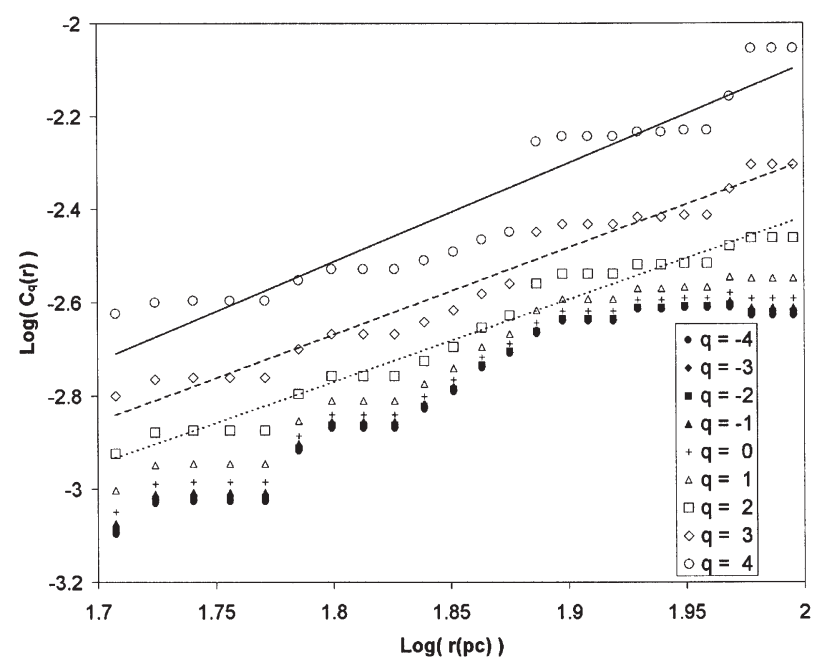

Fig. 5. $C_{q}(r)$ for age group $<20$ Myr old of 126 open clusters.

Figure 4 shows the $\log -\log$ plot of $C_{q}(r)$ for values of $q$ in the range $(-4,4)$ in steps of 1 for the 173 objects in the second sample (age <40 Myr). Again, the slope gives the MinkowskiBouligand dimension for each value of $q$ according to Eq. (8), and we ignore $q \leq 0$ as they produce unphysical results. The slope ranges from $1.87 \pm 0.07$ for $q=2$ to $3.20 \pm 0.17$ for $q=4$. The correlation dimension, $D_{2}$, is $1.87 \pm 0.07, D_{3}=1.23 \pm 0.11$, and $D_{4}=1.07 \pm 0.17$. The correlation coefficient is in the range 0.97-0.98.

Figure 5 shows the $\log -\log$ plot of $C_{q}(r)$ for values of $q$ in the range $(-4,4)$ in steps of 1 for the 126 objects in the third sample (age $<20 \mathrm{Myr}$ ). Again, the slope gives the Minkowski-Bouligand dimension for each value of $q$ according to Eq. (8), and we ignore $q \leq 0$ as they produce unphysical results. The slope ranges from $1.77 \pm 0.07$ for $q=2$ to $2.13 \pm 0.14$ for $q=4$. The correlation dimension, $D_{2}$, is $1.77 \pm 0.07, D_{3}=0.93 \pm 0.08$, and $D_{4}=0.71 \pm 0.14$. The correlation coefficient is in the range $0.95-0.98$.

Finally, Fig. 6 shows the $\log -\log$ plot of $C_{q}(r)$ for values of $q$ in the range $(-4,4)$ in steps of 1 for the 58 objects in the corrected sample (age $<20 \mathrm{Myr}$ and distance $<1.5 \mathrm{kpc}$ ). Again, the slope gives the Minkowski-Bouligand dimension for each value of $q$ according to Eq. (8), and we ignore $q \leq 0$ as they 


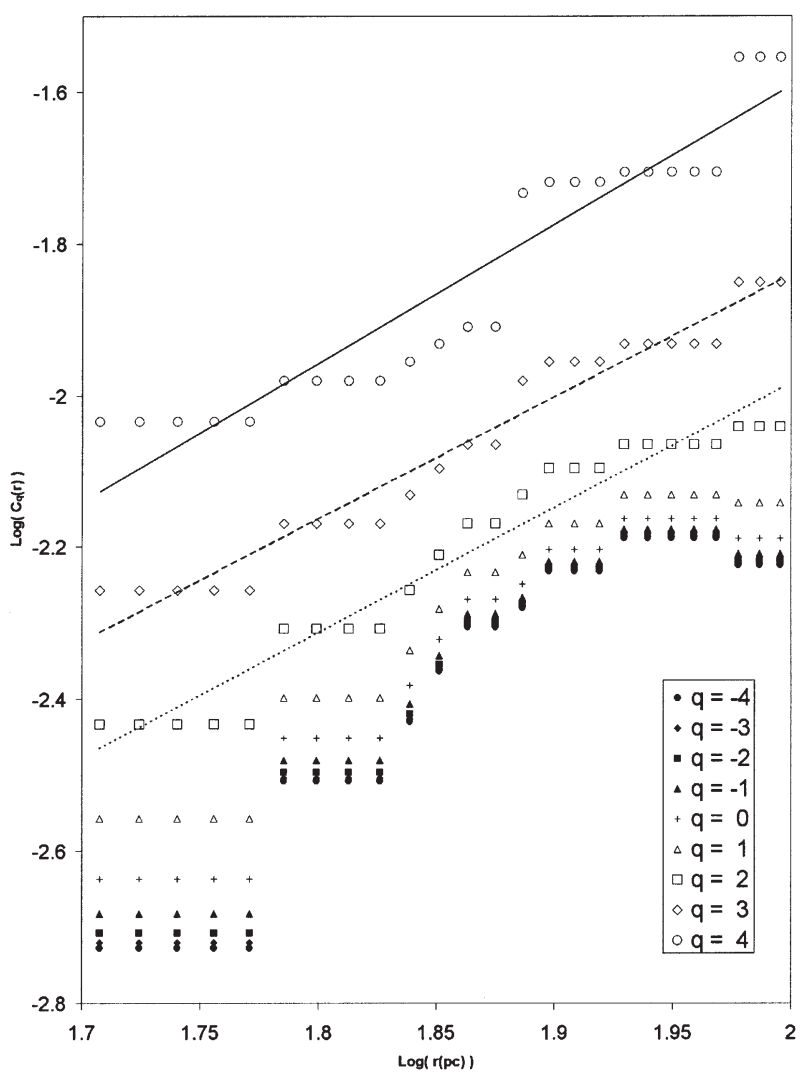

Fig. 6. $C_{q}(r)$ for age group $<20 \mathrm{Myr}$ old of 58 open clusters with heliocentric radial distance $<1.5 \mathrm{kpc}$.

produce unphysical results. The slope ranges from $1.65 \pm 0.08$ for $q=2$ to $1.83 \pm 0.13$ for $q=4$. The correlation dimension, $D_{2}$, is $1.65 \pm 0.08, D_{3}=0.80 \pm 0.07$, and $D_{4}=0.61 \pm 0.13$. The correlation coefficient is in the range $0.95-0.98$.

Figure 7 shows the spectrum of the Minkowski-Bouligand dimensions as a function of $q$ for all the samples. For clusters younger than $60 \mathrm{Myr}, D_{q}$ is in the range 1.13-1.85, and the range is $0.71-1.77$ for young open clusters with ages $<20 \mathrm{Myr}$. The fractal dimension characterises the degree of clumpiness of the young open cluster spatial distribution. As expected, it increases over time because star-forming complexes disintegrate on a relatively short time-scale.

If the young open cluster distribution is homogeneous, $D_{q}$ should not vary with $q$ and it coincides with the Euclidean dimension. However, we find that $D_{q}$ varies with $q$ and that it also appears to change over time.

\section{Comparison with previous work}

Feitzinger \& Galinski (1987) studied the fractal dimension of the distribution of star-forming sites (HII regions) in a sample of 19 spiral galaxies and obtained a mean value of $\langle D\rangle=1.68 \pm$ 0.31 . This agrees very well with the value found for samples younger than $20 \mathrm{Myr}$.

Using archival Hubble Space Telescope images of 10 galaxies, Elmegreen \& Elmegreen (2001) have found that the fractal dimension of star formation in all of the galaxies is $\sim 2.3$. It coincides with the value $2.3 \pm 0.3$ found for the fractal dimension of the interstellar gas by Elmegreen \& Falgarone (1996). Sanchéz et al. (2005) find a value of $2.6 \pm 0.1$ for the fractal dimension in Orion A. Sanchéz et al. (2006) show that 2.6 is roughly consistent with the average properties of the interstellar medium.

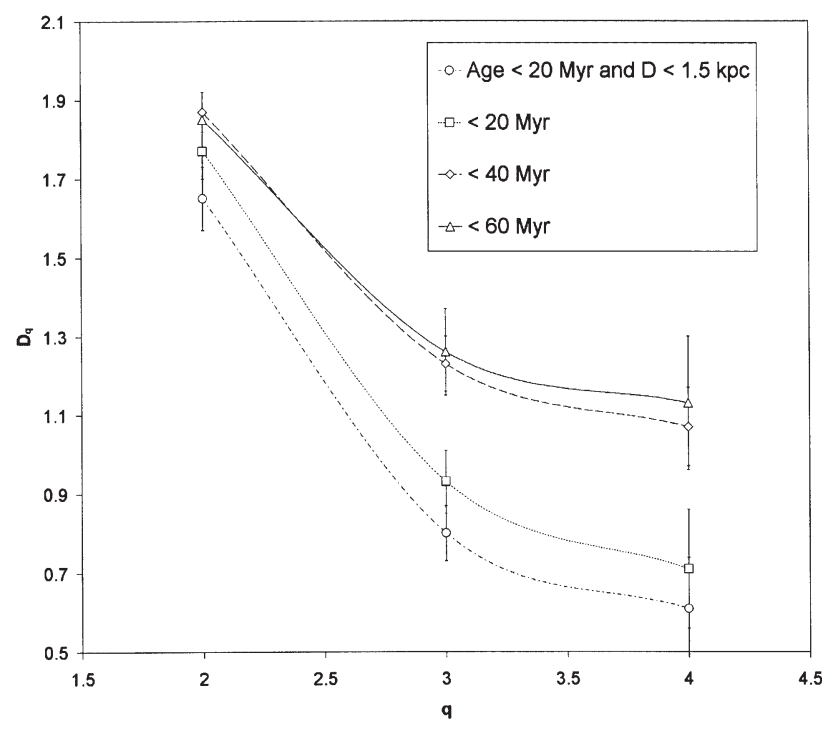

Fig. 7. Spectrum of the Minkowski-Bouligand dimensions as a function of $q$ for the various samples.

Although the technique used to evaluate the fractal dimension in this study is different, the value obtained is consistent with those from other works. In particular, it matches the one found by Feitzinger \& Galinski (1987), which can be interpreted as confirmation of the almost negligible effect of both orbital diffusion and dynamical disruption on the final young open cluster subsample, age $<20 \mathrm{Myr}$ and radial distance $<1.5 \mathrm{kpc}$.

\section{Discussion}

The fractal dimension of a set of points is an important measure of the intrinsic dimensionality of the points. The primary purpose of this paper is to calculate the fractal dimension of the distribution of young open clusters in the Solar Neighbourhood using an empirical approach. In our selection criteria for the final sample we have tried to minimize the effect of dynamical effects, such as open-cluster disruption or radial/azimuthal drift, as well as incompleteness. On the other hand, we are assuming that the objects in the sample are not the result of enhanced star-formation activity (a starburst). For clusters younger than about $40 \mathrm{Myr}$, dynamical effects appear to be less important although $60-70 \%$ of open clusters are destroyed within $20 \mathrm{Myr}$. The surface density of young open clusters remains relatively constant within about $2.5 \mathrm{kpc}$ from the Sun for clusters younger than $40 \mathrm{Myr}$, but it decreases significantly for older clusters.

The results presented above demonstrate that the application of the multifractal formalism to estimate the fractal dimension of the spatial distribution of young open clusters gives unphysical results for $q \leq 0$. The results presented in the previous section clearly show that this method is not valid for studying the structure of the Galactic regions devoid of young clusters (interarm gaps), because the results are unphysical. This seems to point to some artefacts due either to the method used in our calculations or to the actual data sample. The reason for the unusual behaviour observed for $q \leq 0$ appears to be connected to the Galactic spiral structure. As a general rule, no single fractal dimension estimator can be employed with any confidence for a range of $q$ that probe both low- and high-density regions in a point set (Wu et al. 1997). However, results for the clustering of young open clusters $(q>0)$ are both stable and reliable. Ideally, we would expect $D_{q}=2$ for a two-dimensional homogeneous 
and isotropic distribution. We find that for the sample studied, $D_{q}$ varies with $q$. For $q>0$, the value of the Minkowski-Bouligand dimension decreases as $q$ increases, as expected (see Fig. 7).

On the other hand, the behaviour of the spatial distribution of young open clusters is complex; it cannot be characterised by a single exponent (i.e. the fractal dimension). It is a multifractal set, where a whole spectrum of singularity exponents is required in order to provide a reliable geometric description of the distribution. The fractal dimension of young clusters younger than $20 \mathrm{Myr}$ varies from 0.61 to 1.65 , and for clusters younger than $60 \mathrm{Myr}$, it varies from 1.13 to 1.85 . Theoretically, for younger clusters the fractal dimension should be similar to the fractal dimension of the spatial distribution of giant molecular clouds (like 1.68 for HII regions in Feitzinger \& Galinski's paper) then, when the population evolves, the fractal dimension of the surviving clusters should increase as clusters are submitted to a series of processes whose effects are similar to classical diffusion. This is exactly what we observe: it is $1.77 \pm 0.07$ for an age of $20 \mathrm{Myr}$ but for $60 \mathrm{Myr}$ it is $1.85 \pm 0.05$ (for the correlation dimension). The effect is more significant for higher momenta $(q>2)$. One may argue that the significant errors keep this interpretation from being very conclusive, as the values certainly overlap if error intervals are taken into account. This is not, however, true for $D_{3}$ and $D_{4}$ where error intervals do not overlap. Therefore, our results suggest that the fractal dimension increases with age, reflecting the tendency to an increase in the average distance between surviving clusters. If this trend is universal and can be calibrated, then the fractal dimension can be used as an age indicator of extragalactic star-forming regions, especially in spiral galaxies. On the other hand, the fractal dimension can be a powerful parameter to uncover anomalous patterns in star formation associated to non-quiescent star-forming events.

Schmeja \& Klessen (2006) find that the temporal evolution of the clustering parameters shows that star clusters build up from several subclusters and evolve to a more centrally concentrated cluster. This trend has also been found in simulations (Kroupa et al. 2001). In principle, clusters may disappear because of three different processes: (i) subclusters that merge to build up a larger cluster; (ii) evolution towards a kind of cluster remnant; or (iii) full destruction. It should be relatively easy to distinguish between (i) and (ii-iii), but it is rather difficult to decide between (ii) and (iii). If the slope of $C_{q}$ increases it means that the number of centres decrease, but merging does not have the same effect as destruction, since it depends on $q$. Merging only operates on small length-scales within star-forming complexes where the typical intercluster distance is $10-30 \mathrm{pc}$, but destruction should operate on all scales (unless destruction is induced by shocks generated by nearby supernova explosions or very strong stellar winds). If the increase is higher for larger $q$, then it means that merging is dominant; but if the increase is more or less $q$ independent then destruction should be dominant. For the age range 20-40 Myr, the relative variation in $\tau(2)$ is $5 \%, 32 \%$ for $\tau(3)$, and $50 \%$ for $\tau(4)$. However, for the age interval 40-60 Myr, the relative variations are $1 \%, 3 \%$ and $6 \%$ for $q=2,3$, and 4, respectively. This indicates that merging (or proximity-induced destruction, less likely) is dominant for clusters younger than $40 \mathrm{Myr}$ and destruction appears to be dominant in the range 40-60 Myr. On the other hand, simulations suggest that most of the open clusters that are disrupted within a few Myr are small, with less than 50 members. Merging makes larger clusters that are harder to destroy.

Lamers et al. (2005) have found that the present starformation rate in bound clusters is about half the value derived from the study of embedded clusters. This has been interpreted as evidence that half of the clusters in the Solar Neighbourhood become unbound within about $10 \mathrm{Myr}$ of their formation. This destruction rate is even higher than the one discussed here; about $60-70 \%$ of all clusters formed over a period of $20 \mathrm{Myr}$ are being destroyed over the following 20 Myr. Again, the actual decrease in the number of clusters is the result of three processes: merging, full destruction, and evolution to (virtually undetectable) low mass cluster remnants. Therefore, it is quite difficult to provide a conclusive answer to this very important issue.

Acknowledgements. In preparation of this paper, we made use of the NASA Astrophysics Data System and the ASTRO-PH e-print server. This research has made use of the WEBDA database operated at the Institute of Astronomy of the University of Vienna, Austria. We wish to thank the referee, Dr. Bruce G. Elmegreen, for his very helpful report.

\section{References}

Binney, J., \& Tremaine, S. 1987, Galactic Dynamics (Princeton: Princeton University Press) Berghöfer, T.W., Breitschwerdt, D. 2002, A\&A, 390, 299

Bonatto, C., Kerber, L. O., Bica, E., \& Santiago, B. X. 2006, A\&A, 446, 121

Elmegreen, B. G., Efremov, Y., Pudritz, R. E., \& Zinnecker, H. 2000, in Protostars and Planets IV, ed. V. Mannings, A. P. Boss, \& S. S. Russell (University of Arizona Press), 151

Elmegreen, B. G., \& Elmegreen, D. M. 2001, AJ, 121, 1507

Elmegreen, B. G., \& Falgarone, E. 1996, ApJ, 471, 816

Falconer, K. 1990, Fractal Geometry (Chichester: Wiley)

Feitzinger, J. V., \& Galinski, T. 1987, A\&A, 179, 249

de la Fuente Marcos, R., \& de la Fuente Marcos, C. 2004, New Astron., 9, 6

Grassberger, P. 1983, Phys. Lett. A, 97, 227

Grassberger, P., \& Procaccia, I. 1983, Phys. Rev. Lett., 50, 346

Kroupa, P., Aarseth, S. J., \& Hurley, J. 2001, MNRAS, 321, 699

Lamers, H. J. G. L. M., Gieles, M., Bastian, N., et al. 2005, A\&A, 441, 117

Lehner, N., Jenkins, E. B., Gry, C., et al. 2003, ApJ, 595, 858

Maíz Apellániz, J. 2001, ApJ, 560, L83

Mandelbrot, B. B. 1982, The Fractal Geometry of Nature (San Francisco: W. H. Freeman) Mandelbrot, B. B. 1985, Phys. Scr., 32, 257

Mermilliod, J.-C. 1988a, Inform. Bull. CDS, 35, 77

Mermilliod, J.-C. 1988b, in Astronomy from Large Databases, ed. F. Murtagh, \& A. Heck, ESO Conf. and Work. Proc., 41, 419

Mermilliod, J.-C. 1992a, in Astronomy from Large Databases II, ed. A. Heck, \& F. Murtagh, ESO Conf. and Work. Proc., 43, 373

Mermilliod, J.-C. 1992b, Inform. Bull. CDS, 40, 115

Mermilliod, J.-C. 1993, in Databases for Galactic Structure, ed. A. G. D. Philip, B. Hauck, \& A. R. Upgren (Schenectady: L. Davis Press), 27

Mermilliod, J.-C. 1995, in Information and on-line data in Astronomy, ed. D. Egret, \& M. A. Albrecht (Dordrecht: Kluwer), 127

Mermilliod, J.-C. 1996, in The Origins, Evolutions, and Destinies of Binary Stars in Clusters, ed. E. F. Milone, \& J.-C. Mermilliod, ASP Conf. Ser., 90, 475

Paladin, G., \& Vulpiani, A. 1987, Phys. Rep., 156, 147

Paunzen, E., \& Mermilliod, J.-C. 2005, Open Cluster Database, http://www. univie.ac. at/webda

Sánchez, N., Alfaro, E. J., \& Pérez, E. 2005, ApJ, 625, 849

Sánchez, N., Alfaro, E. J., \& Pérez, E. 2006, ApJ, in press, [ArXiv : astro-ph/0512243]

Schmeja, S., \& Klessen, R. S. 2006, A\&A, 449, 151

Sfeir, D. M., Lallement, R., Crifo, F., \& Welsh, B. Y. 1999, A\&A, 346, 785

Wu, K. K. S., Alexander, P., \& Sleath, J. P. 1997, MNRAS, 286, 777

Yadav, J., Bharadwaj, S., Pandey, B., \& Seshadri, T. R. 2005, MNRAS, 364, 601 